\title{
Electromagnetic tracker feasibility in the design of a dental superstructure for edentulous patients
}

\author{
António H.J. Moreira ${ }^{1,2,3}$, Sandro Queirós ${ }^{1}$, Nuno F. Rodrigues ${ }^{1,3}$, ACM Pinho ${ }^{4}$, Jaime C. Fonseca ${ }^{2}$, João L. Vilaça ${ }^{1,3}$ \\ ${ }^{1}$ ICVS/3B's - PT Government Associate Laboratory, School of Health Sciences, Braga/Guimarães, Portugal \\ ${ }^{2}$ Algoritmi Center, School of Engineering, University of Minho, Guimarães, Portugal \\ ${ }^{3}$ DIGARC - Polytechnic Institute of Cávado and Ave, Barcelos, Portugal \\ ${ }^{4}$ Mechanical Department, University of Minho, Guimarães, Portugal
}

\begin{abstract}
The success of the osseointegration concept and the Brånemark protocol is highly associated to the accuracy in the production of an implant-supported prosthesis. One of most critical steps for long-term success of these prosthesis is the accuracy obtained during the impression procedure, which is affected by factors such as the impression material, implant position, angulation and depth. This paper investigates the feasibility of 3D electromagnetic motion tracking systems as an acquisition method for modeling full-arch implant-supported prosthesis. To this extent, we propose an implant acquisition method at the patient mouth and a calibration procedure, based on a 3D electromagnetic tracker that obtains combined measurements of implant's position and angulation, eliminating the use of any impression material. Three calibration algorithms (namely linear interpolation, higher-order polynomial and Hardy multiquadric) were tested to compensate for the electromagnetic tracker distortions introduced by the presence of nearby metals. Moreover, implants from different suppliers were also tested to study its impact on tracking accuracy. The calibration methodology and the algorithms employed proved to implement a suitable strategy for the evaluation of novel dental impression techniques. However, in the particular case of the evaluated electromagnetic tracking system, the order of magnitude of the obtained errors invalidates its use for the full-arch modeling of implant-supported prosthesis.
\end{abstract}

Keywords - edentulous patients; implants measurement; electromagnetic tracker; distortion calibration;

\section{INTRODUCTION}

The medical treatment of partial or complete dental replacement has been significantly improved after the diffusion of osseointegrated implants to support dental prosthesis [1]. Different techniques are available today for manufacturing and assembling these biomechanical systems, typically based on a bridge structure, supported by a series of titanium screws implanted into the edentulous patient's jaw (Fig. 1a) [2].

Many dental techniques describe the use of different polymer printing materials [2] to measure and transfer the tridimensional information (position and orientation) of the patient's implants. Every step in the production of an implant-supported prosthesis influences the fit between the implants and the final prosthesis. One of the most critical steps for the long-term success of the prosthesis is the accuracy during the impression procedure [2], [3], which is affected by factors such as the impression material, implant position, angulation and depth, as well as by difficulties in accessing the oral cavity, the variety of bone properties and its irregular geometrical features [4]-[6].

In general, the microgap (vertical, horizontal or angular, see Fig. 1c) misfit between implant components has been associated with several complications from both mechanical and biological origins. Furthermore, even though singular implant misfits are often imperceptible, the combination of several misfits in different implants may raise complications and compromise the entire prosthesis viability. A common consequence of misfits is screw loosening, which leads to structure instability and implant and/or screw fracture, and soft and/or hard tissue reactions due to increased dental plaque accumulation [7]-[11]. Ultimately, these complications may demand prosthesis repair and, in some cases, its complete replacement for a new prosthesis.

Despite the development of novel and more precise fabrication methods, obtaining an absolute passive fit is practically impossible, especially in complete or partially edentulous patients (Fig. 1b). However, in most cases, small misfit tolerances (under $150 \mu \mathrm{m}$ ) are accepted, given that these do not lead to future implant complications [12], [13].

The main goal of this study is to assess the feasibility of $3 \mathrm{D}$ electromagnetic motion tracking systems as an acquisition method for modeling full-arch implant-supported prosthesis. To this extent, we propose an implant acquisition method at the patient mouth and a calibration procedure, based on a $3 \mathrm{D}$ electromagnetic tracker that obtains combined measurements of implant position and angulation, excluding the need for impression material. The development of this system encompasses three main activities: i) development of a specific tool designed to couple an electromagnetic sensor to an implant; ii) assessment of 3 calibration algorithms to decrease electromagnetic distortions in the tracker readings, namely from nearby metals, such as the titanium in the implants, and iii) evaluate the influence of implants from several manufacturers in the accuracy of the electromagnetic tracker.

The main advantages of this system are the reduction of the acquisition time of the patient jaw model, increased accuracy, reduction of the dependency of the entire procedure on the dentist experience, elimination of the need for physical transportation to the prosthesis centers and, thereby, eliminating the possibility of impression errors due to transport.

This work has been supported by FCT - Fundação para a Ciência e Tecnologia in the scope of the Ph.D. grant SFRH/BD/68270/2010 and the project EXPL/BBB-BMD/2146/2013. 


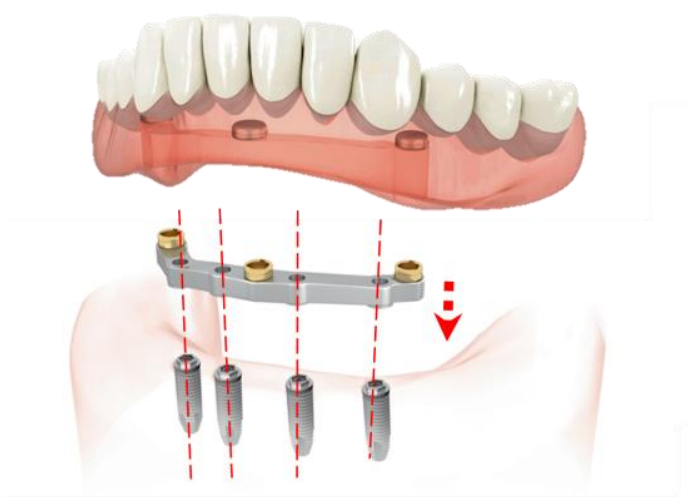

a)

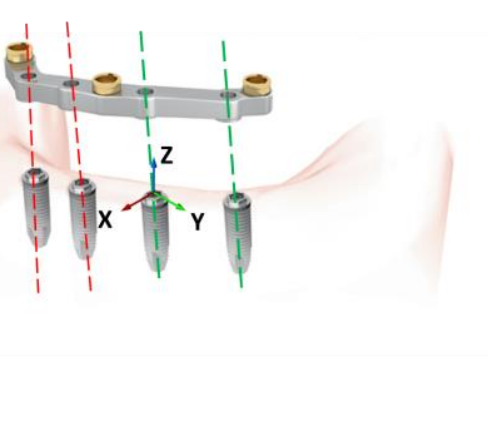

b)

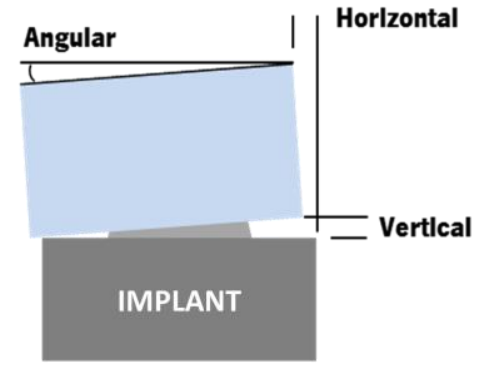

c)

Figure 1 - Osseointegrated implants and support dental prostheses; a) Implants without misfit; b) Implants with partial misfit (in green); c) Different types of microgap misfit (angular, horizontal and vertical).

Furthermore, such a system could be used to validate the manufactured prosthesis against the original virtual model and assure conformance levels before prosthesis placement.

\section{METHODS}

\section{A. Acquisition tool and electromagnetic tracker}

The proposed system requires the development and manufacturing of a miniature customized tool that couples an electromagnetic sensor to a dental implant (Fig. 2a). In this step, a tool was prototyped in Accura Bluestone nanocomposite, to ensure stability and dimensional accuracy, while also being free of electromagnetic interference. This tool was designed to accommodate in one end an abutment with an internal hexagon connection and on the other end an eye-drop sensor from a Polhemus Liberty electromagnetic motion tracking system. Due to the irregular shape of the eye-drop sensor, it was fixed in the tool with an epoxy resin to ensure its stability (Fig. 2b). The tool curved shape design was preferred to allow a more easy acquisition of the innermost implants.

\section{B. System setup}

In order to assess the reliability of the electromagnetic tracker for the implants' position and orientation acquisition, the system was calibrated in a laboratory environment. An industrial 6 DOF robot (ABB IRB140, with $30 \mu \mathrm{m}$ of repeatability) was used to manipulate the tracker tool in a $300 \times 300 \times 300 \mathrm{~mm}$ volume.

Since the robot and the Polhemus system have two different coordinate systems, it is paramount to align the two systems and remove the offsets between them prior to the calibration. In this sense, a rigid registration between the two coordinate spaces is

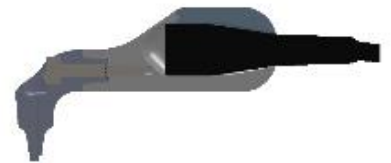

a)

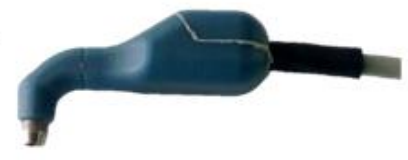

b)
Figure 2 - Prototype tool for implant acquisition; a) cross section; b) final assemble. always performed, prior to the calibration, through an iterative closest point algorithm (ICP) [14].

\section{Calibration methods}

This section presents the employed calibration methods and it's supporting mathematical principles. Suppose that a tracker sensor is moved freely inside a volume forming a cube in the true space. Let $\left\{q_{1}, \ldots, q_{m}\right\}$ denote the true tracker sensor position (measured by the industrial robot) and $\left\{p, \ldots, p_{m}\right\}$ the corresponding tracked measure positions (influenced by the field distortions). For each point $p_{i}$, the location error vector (defined as the spatial difference between the true and measured position) can be computed as $v_{i}=q_{i}-p_{i}$.

The calibration process will thus consist in extracting the information from the known error vectors (from the reference volume) to correct new unknown sensor data. In this sense, a correction vector can be found and applied to the unknown distorted measurement in order to approximate it to its true value. Note that our reference volume consists in a set of scattered points in a 3D volume (due to distortions), thus presenting no structure or order between their relative positions.

\section{1) Linear interpolation:}

Linear interpolation is considered one of the simplest interpolation methods. It corresponds to the estimation of the value of an unknown data by assuming a linear relation between the known values [15]. In the present case, by interpolating the error vectors of neighbor known points (from the reference volume), one is able to estimate the correction to be applied in a new sensor value. Thus, considering the new point $p$, its true position $q$ can be estimated as:

$$
q=p+\sum_{i=1}^{n} w_{i} v_{i}
$$

where $w_{i}$ are normalized weights associated with each neighbor point (in a total of $n$ neighbors) and computed as a function of the distance between point $p$ and its neighbor $\left(p_{i}\right.$ with error vector given by $v_{i}$ ). Note that, due to the scattered nature of our reference volume, a Delaunay triangulation should be firstly applied to find all neighbor points. 


\section{2) Higher-order Polynomial fit:}

In opposition to linear interpolation, higher-order polynomial fitting assumes that the relation between the error vectors, $v_{i}$, and its position, $p_{i}$, can be modelled as an $n$th order polynomial [15], [16]. Considering a polynomial fit of the order $n$, one can fit the error vector as:

$$
v=f(p)=f(x, y, z)=\sum_{j=1}^{N}\left[\begin{array}{l}
c_{x j} \\
c_{y j} \\
c_{z j}
\end{array}\right] x^{s_{j}} y^{t_{j}} z^{u_{j}}
$$

where $c_{x j}, c_{y j}, c_{z j}$ are the polynomial coefficients, $N$ is the number of terms in the polynomial, $N=(n+1)(n+2)(n+$ $3) / 6$, and $s_{j}, t_{j}$ and $u_{j}$ are the nonnegative powers applied to each term such that $0 \leq s_{j}+t_{j}+z_{j} \leq n$ and all permutation of $\left\{s_{j}, t_{j}, z_{j}\right\}$ are unique [17].

The goal is to build the fitting polynomials by determining its coefficients, which corresponds to solve a least-square fit problem for each coordinate $(x, y$ and $z$ ). For example, for the $x$ coordinate, such system of linear equations can be expressed in the matrix form $\boldsymbol{A c}=\boldsymbol{v}$ as:

$$
\left[\begin{array}{ccccc}
a_{1,1} & \cdots & a_{1, j} & \cdots & a_{1, N} \\
\vdots & \vdots & \vdots & \vdots & \vdots \\
a_{i, 1} & \cdots & a_{i, j} & \cdots & a_{i, N} \\
\vdots & \vdots & \vdots & \vdots & \vdots \\
a_{m, 1} & \cdots & a_{m, j} & \cdots & a_{m, N}
\end{array}\right]\left[\begin{array}{c}
c_{x, 1} \\
\vdots \\
c_{x, j} \\
\vdots \\
c_{x, N}
\end{array}\right]=\left[\begin{array}{c}
v_{1} \\
\vdots \\
v_{i} \\
\vdots \\
v_{m}
\end{array}\right]
$$

where $a_{i, j}=x_{i}^{s_{j}} y_{i}^{t_{j}} z_{i}^{u_{j}}$. After solving such system for each coordinate, the corrected point $q$ of a new sensor data point $p$ is given by $q=p+v$, where $v$ is the correction vector found by multiplying matrix A with the coefficients vector for each coordinate.

\section{3) Hardy Multiquadric method (HMQ):}

Hardy's multiquadric (HMQ) interpolation algorithm [17][19] is a global interpolation method that comes from the field of topography, with excellent results over scattered data in one or more dimensions. According to its formulation, the true value of a sensor data point, $q$, is given by:

$$
q=f(p)=\sum_{j=1}^{m} \alpha_{j} \cdot \omega_{j}(p)
$$

where

$$
\omega_{j}(p)=\sqrt{\left(p-p_{j}\right)^{2}+R^{2}}
$$

and $\mathrm{R}$ is the shape parameter controlling the interpolation and $\alpha_{j}$ are the expansion coefficient vectors ( $x, y$ and $z$ ).

In order to correct new sensor values, the unknown expansion coefficients need to be determined. To this end, the reference volume points, $q_{i}$, and their corresponding sensor points, $p_{i}$, can be used to create a system of linear equations, easily expressed in the matrix form $\boldsymbol{W} \boldsymbol{A}=\boldsymbol{q}$ as:

$$
\left[\begin{array}{ccccc}
\omega_{1}\left(p_{1}\right) & \cdots & \omega_{j}\left(p_{1}\right) & \cdots & \omega_{m}\left(p_{1}\right) \\
\vdots & \vdots & \vdots & \vdots & \vdots \\
\omega_{1}\left(p_{i}\right) & \cdots & \omega_{j}\left(p_{i}\right) & \cdots & \omega_{m}\left(p_{i}\right) \\
\vdots & \vdots & \vdots & \vdots & \vdots \\
\omega_{1}\left(p_{m}\right) & \cdots & \omega_{j}\left(p_{m}\right) & \cdots & \omega_{m}\left(p_{m}\right)
\end{array}\right]\left[\begin{array}{c}
\alpha_{1} \\
\vdots \\
\alpha_{j} \\
\vdots \\
\alpha_{m}
\end{array}\right]=\left[\begin{array}{c}
q_{1} \\
\vdots \\
q_{i} \\
\vdots \\
q_{m}
\end{array}\right]
$$

By solving such system, the required expansion coefficients are found and any uncorrected sensor value, $p$, can be compensated using equation (4).

\section{EXPERIMENTS}

In order to assess the performance of the different calibration methods, two types of evaluations were performed: first, a technical test to analyze the accuracy of the calibration algorithms by adding Gaussian noise to known points; and secondly, using real validation points extracted from the acquired volume to corroborate the previous results. Finally, a third test was performed to assess the implants' material influence in the accuracy of the proposed measurement system.

In the experiments, the proposed tool (Section II.A) was fixed at an extremity of a carbon-fiber $10 \times 10 \mathrm{~mm}$ square tube with $600 \mathrm{~mm}$ length, and with the opposite extremity fixed on the robot tip (Fig. 3). The described system was used to acquire samples inside the $300 \times 300 \times 300 \mathrm{~mm}$ volume (with $30 \mathrm{~mm}$ step, in a total of 1331 points) to be used as references for the calibration process. For each point of the volume, 1000 samples from the electromagnetic tracker were acquired and averaged without any implant on the tip of the tool. Complementing the volume points, 30 validation points were also acquired to assess the calibration performance.

\section{A. Calibration performance}

Evaluation of the calibration performance was carried out to analyze which algorithm is the most suitable to compensate the electromagnetic distortions.

1) Gaussian noise: In this test, a known Gaussian noise (with a sigma value of $50 \mathrm{~mm}$ and a maximum value of $10 \mathrm{~mm}$ ) was added to the reference value in the $Z$ direction, based on the $\mathrm{X}$ and $\mathrm{Y}$ coordinate values (Fig. 4).

Using the calibration methods presented in Section II.C, the average error and its standard deviation was computed for each method, using 30 random validation points. In the case of higher-order polynomial fit, an order of 3 to 5 was tested.

\section{2) Real sensor data:}

In this test, the 30 validation points initially acquired were used to analyze the errors, in a real case scenario, before and

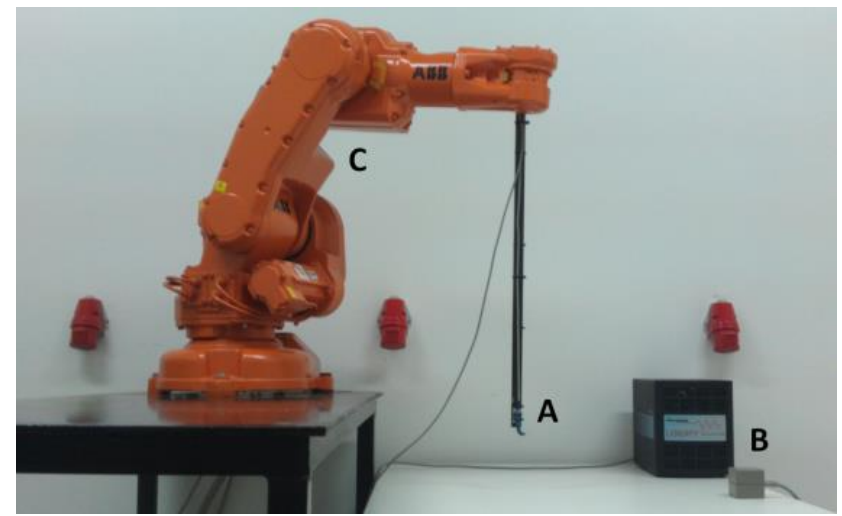

Figure 3- System setup - A) prototype tool; B) Polhemus electromagnetic tracker and $C$ ) robot $A B B$ IRB140. 
after applying each algorithm. As in the first test, both average and standard deviation errors were computed.

\section{B. Implants' material influence}

Evaluating the implants' influence is fundamental to determine if the electromagnetic tracker is capable of measuring the tridimensional location accurately to allow the design of the dental superstructure.

In order to study such influence, three implants from different manufacturers (DIO Implant, Nobel Biocare and Straumann) were attached to the tool and moved inside the reference volume. For each implant, 30 validation points were acquired (in the same positions as the initial ones without implant). The calibration methods were then applied and the resulting discrepancies from the ground truth (robot points) were assessed.

\section{RESULTS AND DISCUSSION}

In regard to the calibration performance for a volume distorted by a Gaussian noise, results for the mean error and standard deviation in each axis, and their combined magnitude, are summarized in Table I.

Table I - Calibration performance for each algorithm (mean error and standard deviation for each axis and combined magnitude) in the simulated case with added Gaussian noise.

\begin{tabular}{|c|c|c|c|c|}
\hline \multirow{2}{*}{$\begin{array}{c}\text { Calibration } \\
\text { Methods }\end{array}$} & \multicolumn{4}{|c|}{ Error $(\mathbf{m m}, \boldsymbol{\mu} \pm \boldsymbol{\sigma})$} \\
\cline { 2 - 5 } & Magnitude & $\boldsymbol{X}$ & $\boldsymbol{Y}$ & $\boldsymbol{Z}$ \\
\hline None & $1.82 \pm 2.42$ & $0 \pm 0$ & $0 \pm 0$ & $1.82 \pm 2.42$ \\
\hline $\begin{array}{c}\text { Linear } \\
\text { Interpolation }\end{array}$ & $0.10 \pm 0.11$ & $0 \pm 0$ & $0 \pm 0$ & $0.10 \pm 0.11$ \\
\hline $\begin{array}{c}3^{\text {rd }} \text { order } \\
\text { polynomial }\end{array}$ & $1.23 \pm 1.04$ & $0 \pm 0$ & $0 \pm 0$ & $1.23 \pm 1.04$ \\
\hline $\begin{array}{c}4^{\text {th }} \text { order } \\
\text { polynomial }\end{array}$ & $0.71 \pm 0.54$ & $0 \pm 0$ & $0 \pm 0$ & $0.71 \pm 0.54$ \\
\hline $\begin{array}{c}5^{\text {th }} \text { order } \\
\text { polynomial }\end{array}$ & $0.71 \pm 0.54$ & $0 \pm 0$ & $0 \pm 0$ & $0.71 \pm 0.54$ \\
\hline $\begin{array}{c}\text { Hardy } \\
\text { multiquadric }\end{array}$ & $0.03 \pm 0.03$ & $0.02 \pm 0.02$ & $0.01 \pm 0.01$ & $0.02 \pm 0.02$ \\
\hline
\end{tabular}

All algorithms presented significant improvements when compared to the initial error (no calibration). Nevertheless, there were clear differences between methodologies. On the one hand, the polynomial interpolation did not perform well due to its global interpolation nature, which have the tendency to globally approximate the Gaussian distortion shape and therefore fails in the Gaussian peak region and in the borders. On the other hand, both linear and HMQ interpolation methods achieved the best results. Although the linear method has a local behavior, it works well for regular shapes as long as the acquired samples are well spread across the volume (which is the case of the current experiment). Regarding Hardy multiquadric interpolation, even though it is the best among all used algorithms, it also introduces error in the $\mathrm{X}$ and $\mathrm{Y}$ axes (in contrast with the other algorithms). This is related to its weighting average function that takes into account all acquired points for the calibration. Nevertheless, its ability to approximate even more complex shapes makes it one of the most capable algorithms to reduce electromagnetic distortions.

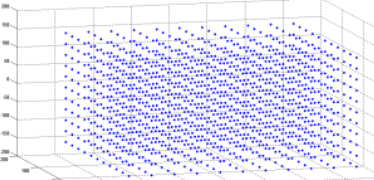

a)
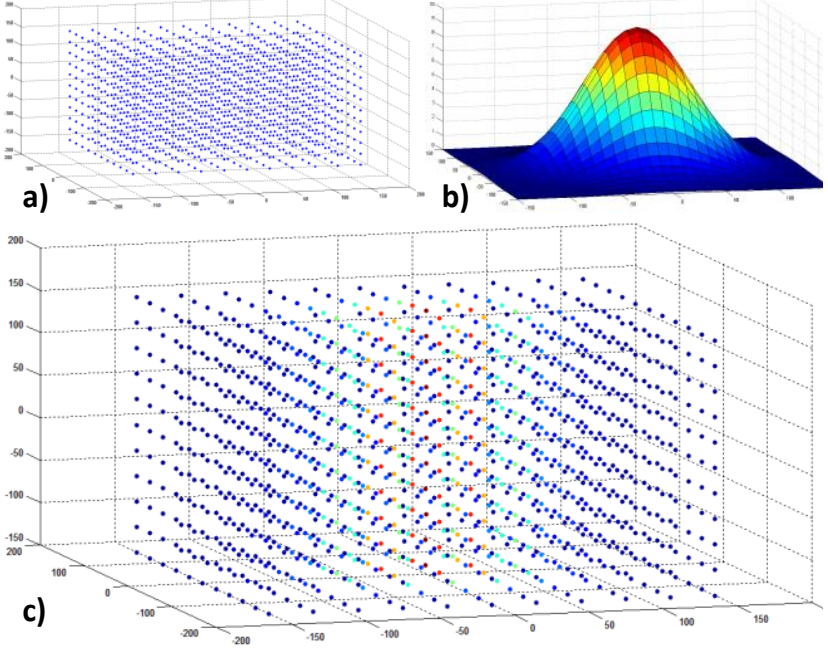

Figure $4-a)$ Reference volume according to the robots position

b) Gaussian noise to be added in the Z component; $c$ ) Resulting distorted samples to be used for calibration assessment.

In regard to the calibration performance for the real data (30 validation points acquired with the prototype tool), results for the mean error and standard deviation in each axis, and their combined magnitude, are summarized in Table II.

Table II - Calibration performance for each algorithm (mean error and standard deviation for each axis and combined magnitude) with the real data acquired with the Polhemus tracker system.

\begin{tabular}{|c|c|c|c|c|}
\hline \multirow{2}{*}{$\begin{array}{c}\text { Calibration } \\
\text { Methods }\end{array}$} & \multicolumn{4}{|c|}{ Error $(\mathbf{m m}, \boldsymbol{\mu} \pm \boldsymbol{\sigma})$} \\
\cline { 2 - 5 } & Magnitude & $\boldsymbol{X}$ & $\boldsymbol{Y}$ & $\boldsymbol{Z}$ \\
\hline None & $4.03 \pm 2.44$ & $1.11 \pm 0.99$ & $2.47 \pm 2.20$ & $2.27 \pm 2.01$ \\
\hline $\begin{array}{c}\text { Linear } \\
\text { Interpolation }\end{array}$ & $0.50 \pm 0.23$ & $0.13 \pm 0.12$ & $0.45 \pm 0.25$ & $0.07 \pm 0.06$ \\
\hline $\begin{array}{c}3^{\text {rd }} \text { order } \\
\text { polynomial }\end{array}$ & $0.71 \pm 0.35$ & $0.19 \pm 0.14$ & $0.56 \pm 0.35$ & $0.28 \pm 0.25$ \\
\hline $\begin{array}{c}4^{\text {th }} \text { order } \\
\text { polynomial }\end{array}$ & $0.56 \pm 0.30$ & $0.16 \pm 0.13$ & $0.49 \pm 0.30$ & $0.13 \pm 0.10$ \\
\hline $\begin{array}{c}5^{\text {th }} \text { order } \\
\text { polynomial }\end{array}$ & $0.53 \pm 0.26$ & $0.14 \pm 0.13$ & $0.47 \pm 0.27$ & $0.12 \pm 0.08$ \\
\hline $\begin{array}{c}\text { Hardy } \\
\text { multiquadric }\end{array}$ & $0.53 \pm 0.25$ & $0.15 \pm 0.14$ & $0.47 \pm 0.26$ & $0.11 \pm 0.09$ \\
\hline
\end{tabular}

As in the previous case, all calibration algorithms lead to improvements against the initial error (no calibration) with close values between each other. In this case, the local interpolation performed best, by a small margin, when compared to Hardy multiquadric interpolation. This is probably related to the relatively smoother distortion along the axes of the electromagnetic tracker, with only a small fraction of these points with higher distortion effects (Fig. 5). Overall, a higher stability and consistency of the results is observed with the HMQ calibration

In regard to the implants' material influence in the electromagnetic tracker accuracy, results for the mean error and standard deviation for each implant are summarized in Table III. 


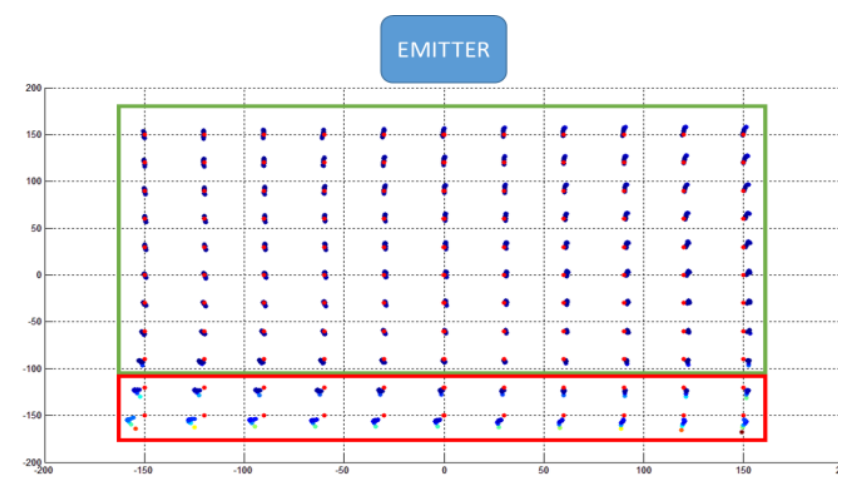

Figure 5 - Electromagnetic distortion in the XY plane using the prototype tool (red zone: high distortion; green zone: low distortion).

Table III - Calibration performance for each algorithm (mean magnitude error and standard deviation) when different implants are introduced in the prototype tool.

\begin{tabular}{|c|c|c|c|c|}
\hline Calibration & \multicolumn{4}{|c|}{ Error $(\mathbf{m m}, \boldsymbol{\mu} \pm \boldsymbol{\sigma})$} \\
\cline { 2 - 5 } Methods & No Implant & Implant 1 & Implant 2 & Implant 3 \\
\hline None & $4.03 \pm 2.44$ & $4.12 \pm 2.29$ & $3.97 \pm 2.42$ & $3.97 \pm 2.42$ \\
\hline $\begin{array}{c}\text { Linear } \\
\text { Interpolation }\end{array}$ & $0.50 \pm 0.23$ & $0.58 \pm 0.26$ & $0.54 \pm 0.28$ & $0.53 \pm 0.28$ \\
\hline $\begin{array}{c}3^{\text {rd }} \text { order } \\
\text { polynomial }\end{array}$ & $0.71 \pm 0.35$ & $0.71 \pm 0.32$ & $0.75 \pm 0.38$ & $0.75 \pm 0.38$ \\
\hline $\begin{array}{c}4^{\text {th }} \text { order } \\
\text { polynomial }\end{array}$ & $0.56 \pm 0.30$ & $0.59 \pm 0.26$ & $0.61 \pm 0.33$ & $0.60 \pm 0.33$ \\
\hline $\begin{array}{c}5^{\text {th }} \text { order } \\
\text { polynomial }\end{array}$ & $0.53 \pm 0.26$ & $0.53 \pm 0.25$ & $0.58 \pm 0.29$ & $0.58 \pm 0.29$ \\
\hline $\begin{array}{c}\text { Hardy } \\
\text { multiquadric }\end{array}$ & $0.53 \pm 0.25$ & $0.55 \pm 0.24$ & $0.59 \pm 0.29$ & $0.58 \pm 0.29$ \\
\hline
\end{tabular}

In the last test, all calibration algorithms performed nearly identical in the presence of the different titanium implants. Analyzing the two most accurate algorithms (the linear interpolation and the Hardy multiquadric method) errors between $20 \mu \mathrm{m}$ and $80 \mu \mathrm{m}$ were observed. Concerning interimplant errors, the range stands between $10 \mu \mathrm{m}$ and $50 \mu \mathrm{m}$. These errors are of much lower magnitude when compared to the tracker system errors (Table II), leading to the conclusion that the major source of possible misfits are related to electromagnetic distortions from nearby objects.

\section{CONCLUSION}

This work presents a study to assess the feasibility of 3D electromagnetic tracking systems as an acquisition method of implant position for modeling full-arch implant-supported prosthesis. The main advantages of this system are the reduction of the acquisition time of the patient jaw model, increased accuracy, and reduction of the dependency of the entire procedure on the dentist experience.

To this extent, three calibration algorithms (linear interpolation, higher-order polynomial and Hardy multiquadric) were tested to compensate for the electromagnetic tracker distortions. Moreover, implants form different suppliers were also tested to study its impact on tracking accuracy.

The calibration methodology and the algorithms employed proved to implement a suitable strategy for the evaluation of novel dental impression techniques. However, in the particular case of the evaluated electromagnetic tracking system, Polhemus Liberty, the order of magnitude of the obtained errors invalidates its use for the full-arch modeling of implantsupported prosthesis. The study showed that this impossibility relies not in the calibration procedure nor on other postprocessing techniques, but rather on the physical limitations of the tracking device, in particular its high sensitivity to magnetic interferences. Furthermore, since most electromagnetic tracking devices, able to cope with a volume greater than $27.000 \mathrm{~cm}^{3}$, use similar principles to the tested one, one is strongly convinced by this study that these kind of systems may not be adequate for medical applications which demand error margins under $150 \mu \mathrm{m}$, especially if in the presence of ferromagnetic materials. Nevertheless, we believe there is an opportunity for this kind of tracking systems to be used in other medical applications.

Our current research is focused on the identification of different principles and sensors for the accurate acquisition of implant positions and angulations, in particular using specific mechanical systems, instrumented with optical sensors for accurate measurement.

\section{REFERENCES}

[1] P.-I. Branemark, G. A. Zarb, T. Albrektsson, and H. M. Rosen, "Tissue-integrated prostheses. osseointegration in clinical dentistry," Plast. Reconstr. Surg., vol. 77, no. 3, pp. 496-497, 1986.

[2] H. Lee, J. S. So, J. L. Hochstedler, and C. Ercoli, "The accuracy of implant impressions: a systematic review.," $J$. Prosthet. Dent., vol. 100, no. 4, pp. 285-91, Oct. 2008.

[3] I. A. Al-Bakri, D. Hussey, and W. M. Al-Omari, "The dimensional accuracy of four impression techniques with the use of addition silicone impression materials.," $J$. Clin. Dent., vol. 18, no. 2, pp. 29-33, Jan. 2007.

[4] W. G. Assuncao, H. G. Filho, and O. Zaniquelli, "Evaluation of transfer impressions for osseointegrated implants at various angulations.," Implant Dent., vol. 13, no. 4, pp. 358-66, Dec. 2004.

[5] W. G. Assunção, R. C. Britto, V. A. Ricardo Barão, J. A. Delben, and P. H. dos Santos, "Evaluation of impression accuracy for implant at various angulations.," Implant Dent., vol. 19, no. 2, pp. 167-74, Apr. 2010.

[6] R. Sorrentino, E. F. Gherlone, G. Calesini, and F. Zarone, "Effect of implant angulation, connection length, and impression material on the dimensional accuracy of implant impressions: an in vitro comparative study.," Clin. Implant Dent. Relat. Res., vol. 12 Suppl 1, pp. e6376, May 2010.

[7] C. J. Goodacre, G. Bernal, K. Rungcharassaeng, and J. Y. K. Kan, "Clinical complications in fixed prosthodontics.," J. Prosthet. Dent., vol. 90, no. 1, pp. 31-41, Jul. 2003.

[8] T.-M. Wang, L.-J. Leu, J. Wang, and L.-D. Lin, "Effects of prosthesis materials and prosthesis splinting on periimplant bone stress around implants in poor-quality bone: a numeric analysis.," Int. J. Oral Maxillofac. Implants, vol. 17, no. 2, pp. 231-7, 2002.

[9] S. Sahin, M. C. Cehreli, and E. Yalçin, "The influence of functional forces on the biomechanics of implant- 
supported prostheses--a review.," J. Dent., vol. 30, no. 78, pp. 271-82, 2002.

[10] S. E. Eckert, S. J. Meraw, E. Cal, and R. K. Ow, “Analysis of incidence and associated factors with fractured implants: a retrospective study.," Int. J. Oral Maxillofac. Implants, vol. 15, no. 5, pp. 662-7, 2000.

[11] A. Eliasson, A. Wennerberg, A. Johansson, A. Ortorp, and T. Jemt, "The precision of fit of milled titanium implant frameworks (I-Bridge) in the edentulous jaw.," Clin. Implant Dent. Relat. Res., vol. 12, no. 2, pp. 81-90, Jun. 2010.

[12] S. Sahin and M. C. Cehreli, "The significance of passive framework fit in implant prosthodontics: current status.," Implant Dent., vol. 10, no. 2, pp. 85-92, Jan. 2001.

[13] J. Y. Kan, K. Rungcharassaeng, K. Bohsali, C. J. Goodacre, and B. R. Lang, "Clinical methods for evaluating implant framework fit.," J. Prosthet. Dent., vol. 81, no. 1, pp. 7-13, Jan. 1999.

[14] S. Rusinkiewicz and M. Levoy, "Efficient variants of the ICP algorithm," in Proceedings Third International
Conference on 3-D Digital Imaging and Modeling, 2001, pp. $145-152$.

[15] S. T. Bryson, "Measurement and calibration of static distortion of position data from 3D trackers," in SPIE/IS\&T 1992 Symposium on Electronic Imaging: Science and Technology, 1992, pp. 244-255.

[16] V. Kindratenko, "Calibration of electromagnetic tracking devices," Virtual Real., vol. 4, no. 2, pp. 139-150, Jun. 1999.

[17] V. V Kindratenko, "A survey of electromagnetic position tracker calibration techniques," Virtual Real., vol. 5, no. 3, pp. 169-182, 2000.

[18] R. L. Hardy, "Multiquadric equations of topography and other irregular surfaces," J. Geophys. Res., vol. 76, no. 8, pp. 1905-1915, Mar. 1971.

[19] G. Zachmann, "Distortion correction of magnetic fields for position tracking," in Proceedings Computer Graphics International, 1997, pp. 213-220, 